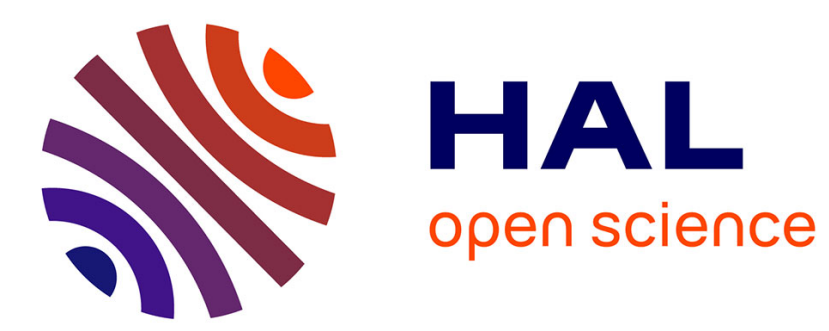

\title{
Envisager le jeu vidéo comme une filière des industries culturelles et médiatiques
}

\author{
Lucien Perticoz
}

\section{To cite this version:}

Lucien Perticoz. Envisager le jeu vidéo comme une filière des industries culturelles et médiatiques.

Les Enjeux de l'information et de la communication, 2011. hal-01792581

\section{HAL Id: hal-01792581 \\ https://univ-lyon3.hal.science/hal-01792581}

Submitted on 31 May 2018

HAL is a multi-disciplinary open access archive for the deposit and dissemination of scientific research documents, whether they are published or not. The documents may come from teaching and research institutions in France or abroad, or from public or private research centers.
L'archive ouverte pluridisciplinaire HAL, est destinée au dépôt et à la diffusion de documents scientifiques de niveau recherche, publiés ou non, émanant des établissements d'enseignement et de recherche français ou étrangers, des laboratoires publics ou privés. 


\title{
ENVISAGER LE JEU VIDÉO COMME UNE FILIÈRE DES INDUSTRIES CULTURELLES ET MÉDIATIQUES
}

Lucien Perticoz

\author{
GRESEC | «Les Enjeux de l'information et de la communication »
}

2011/1 n 12/1 | pages 125 à 142

Article disponible en ligne à l'adresse :

https://www.cairn.info/revue-les-enjeux-de-l-information-et-de-lacommunication-2011-1-page-125.htm

\section{Pour citer cet article :}

Lucien Perticoz, «Envisager le jeu vidéo comme une filière des industries culturelles et médiatiques », Les Enjeux de l'information et de la communication 2011/1 ( ${ }^{\circ}$ $12 / 1)$, p. 125-142.

Distribution électronique Cairn.info pour GRESEC.

(C) GRESEC. Tous droits réservés pour tous pays.

La reproduction ou représentation de cet article, notamment par photocopie, n'est autorisée que dans les limites des conditions générales d'utilisation du site ou, le cas échéant, des conditions générales de la licence souscrite par votre établissement. Toute autre reproduction ou représentation, en tout ou partie, sous quelque forme et de quelque manière que ce soit, est interdite sauf accord préalable et écrit de l'éditeur, en dehors des cas prévus par la législation en vigueur en France. Il est précisé que son stockage dans une base de données est également interdit. 


\section{Envisager le jeu vidéo comme une filière des industries culfurelles et médiatiques}

Article inédit. Mis en ligne le 15 avril 2011.

\section{Lucien Perticoz}

Lucien Perticoz est docteur en sciences de l'information et de la communication et a soutenu sa thèse au sein $d u$ Gresec (Groupe de recherche sur les enjeux de la communication) à Échirolles, sous la direction de Bernard Miège. Ses recherches portent principalement sur les articulations entre l'évolution des pratiques culturelles médiatiques et les mutations au sein des industries culturelles. Son travail doctoral est plus particulièrement axé sur la pervasivité de l'écoute de musique enregistrée permise par la numérisation et ses implications socioéconomiques au niveau de la filière phonographique.

Plan

Introduction

Un loisir et un secteur économique en voie de légitimation artistique

La théorie des industries culturelles comme grille d'analyse de la filière du jeu vidéo...

... L'étude de la filière du jeu vidéo pour enrichir la théorie des industries culturelles

Conclusion

Références bibliographiques

\section{Résumé}

Cet article a pour objectif de tracer les grandes lignes d'une approche interdisciplinaire de l'industrie du jeu vidéo. En mobilisant les travaux sur les mutations des industries culturelles et médiatiques (ICM), nous entendons proposer un cadre théorique nous permettant d'en appréhender les modalités de fonctionnement socio-économiques. Nous verrons dans quelle mesure le jeu vidéo peut, tout comme le cinéma ou la musique enregistrée, être considéré comme une filière des ICM. Dans le même temps, nous appuyant sur les spécificités socio-économiques du secteur vidéoludique, nous entamerons une discussion théorique concernant l'évolution des modalités de production, de distribution et de valorisation marchande existant au sein des ICM.

Mots-clés : jeux vidéo, socio-économie, mutations, industries culturelles et médiatiques, filière, numérique.

\section{Abstract}

This article aims to propose an interdisciplinary approach of the video games industry. Using the researches about changes in cultural industries, we want to establish a theoretical framework which helps us to understand its socio-economic procedures. As the movie and the music industry, we'll see how video game may also be considered as a cultural industries sector. Taking in consideration the socio-economic specificities of the video games industry, we will start a theoretical discussion concerning the evolution of the existing procedures of production, distribution and economical valorization.

Keywords: video games, socio-economy, changes, cultural industries, industrial sector, digital.

\section{Resumen}

El objetivo de este artículo es de proponer un enfoque interdisciplinario de la industria del videojuego. Utilizando los trabajos sobre los cambios de las industrias culturales y de información, queremos establecer un marco teórico que nos permite comprender sus modalidades de funcionamiento socioeconómicas. De la misma manera que para las 
industrias cinematográficas y fonográficas, veremos en qué medida el videojuego puede también considerarse como un sector de las industrias culturales. En el mismo tiempo, teniendo en cuenta las especificidades socioeconómicas de este sector, entablaremos un debate teórico relativa a la evolución de las modalidades de producción, distribución y valorización comercial que existen ahora en las industrias de la cultura.

Palabras clave: videojuegos, socio-economía, cambios, industrias culturales y de información, sector industrial, numérico.

\section{INTRODUCTION}

En première lecture, le titre du présent article peut paraître relever d'un certain sens commun, voire de l'évidence. Pour nombre d'acteurs, qu'ils soient politiques, économiques ou académiques, il ne fait en effet plus guère de doute que le secteur du jeu vidéo doive être considéré comme une filière des ICM, au même titre que le cinéma, l'édition ou l'industrie phonographique. Qu'il s'agisse des processus de production ou de l'importance économique qu'elle représente (plus de 50 milliards d'euros de chiffre d'affaires dans le monde en $2009^{1}$ ), cette filière semble avoir d'ores et déjà acquis une relative maturité. Pourtant, si la communauté scientifique n'a plus de réelles réticences à l'appréhender comme un objet de recherche légitime, le jeu vidéo n’a été que très peu interrogé à la lumière des travaux en SIC sur les mutations des ICM. Les recherches francophones menées sont de plus en plus nombreuses mais se placent principalement sur le plan de l'analyse des effets de la pratique vidéoludique, de la construction identitaire des gamers, des processus narratifs propres au jeu vidéo, de ses rapports avec la culture populaire ou encore de la dimension idéologique de certains titres (Blanchet, 2010). Alors que des auteurs anglo-saxons se sont intéressés à l'industrie du jeu vidéo en convoquant les travaux s'inscrivant dans le cadre de l'économie politique de la communication (Dyer-Witheford \& de Peuter, 2009 ; Kline \& alii, 2003), ce type d'approche est très loin d'être développé en France.

Certains auteurs ont toutefois été amenés à évoquer dans leurs productions les apports des recherches menées sur les mutations des ICM. C'est notamment le cas de Genvo qui a mobilisé, dans le cadre de ses travaux sur le game design de jeux vidéo, la notion de modèles socio-économiques développée par Miège et d'autres auteurs (Genvo, 2006, 2009). Le fait qu'il convoque les notions de modèles éditorial et de flot développées par ces derniers laisse entendre que le jeu vidéo doit être, selon lui, considéré comme une filière à part entière des ICM. Toutefois, le travail de démonstration théorique n'est pas véritablement entrepris et l'auteur ne reprend pas les principales spécificités des ICM (industries de prototypes, incertitude sur les valeurs d'usage des biens produits, dialectique du tube et du catalogue, etc.) pour appuyer son propos. Si l'éclairage théorique est esquissé, il n'est finalement que très peu développé.

A l'autre extrémité, des auteurs qui mènent depuis plus de trente ans des recherches sur les mutations des ICM paraissent encore réticents à l'idée de considérer le jeu vidéo comme une industrie culturelle au sens plein et théoriquement validé du terme. Certains préfèrent ainsi l'inclure dans un ensemble aux contours plus flous qui serait celui des industries créatives (Tremblay, 2008). Selon Tremblay, il ressort que présenter le jeu vidéo comme

\footnotetext{
${ }^{1}$ Source : IDATE, « L'industrie de jeux vidéo pèse 50,4 milliards EUR en 2010 », URL : http://blog.idate.fr/?p=181 (consulté le 05/04/2011).
} 
une nouvelle filière des industries culturelles serait loin d'être scientifiquement fondé et resterait sujet à caution. Néanmoins, à la lecture des arguments avancés, ce pas de côté théorique semble finalement plus être une manière d'exclure de fait le jeu vidéo du champ des ICM, alors même que les spécificités de l'industrie vidéoludique plaident plutôt pour son inclusion. Sans aller jusqu'à parler d'ignorance réciproque entre ces deux courants de la recherche, force est toutefois de constater que les échanges entre eux n'ont finalement été ni très nombreux ni particulièrement encouragés.

Il apparaît pourtant qu'il y aurait un intérêt théorique des plus féconds à interroger le secteur du jeu vidéo à la lumière des acquis les plus sûrs des différentes recherches menées sur les mutations des ICM. Nous voyons un double bénéfice à promouvoir cette approche. Elle doit tout d'abord nous permettre de montrer en quoi les spécificités du jeu vidéo font de celui-ci une filière des industries culturelles à part entière. Ensuite, les particularités propres à cette dernière viennent éclairer d'un jour nouveau ces acquis. Tout en les confirmant pour partie, elles nous obligent aussi à venir les compléter et à les réinterroger. De ce fait, c'est bien à un enrichissement à différents niveaux (modes de production, de distribution, de valorisation marchande et évolutions des pratiques culturelles médiatiques) des théories en question que l'étude du secteur du jeu vidéo doit permettre de contribuer.

Nous entendons ainsi développer notre démarche en trois temps. Nous verrons dans une première partie comment le jeu vidéo a réussi à acquérir une reconnaissance aussi bien institutionnelle, économique qu'artistique, ces trois niveaux étant intimement liés entre eux. Nous entreprendrons dans un second temps une confrontation entre les spécificités du jeu vidéo et les théories des mutations des ICM. L'objectif sera de démontrer l'intérêt et la pertinence d'appréhender les jeux vidéo comme une des filières des industries culturelles. Ce travail de cadrage théorique effectué, nous montrerons dans une troisième partie en quoi l'étude du champ du jeu vidéo ouvre des perspectives des plus heuristiques pour la recherche. Nous considérons ainsi qu'elle favoriserait un enrichissement et un affinement des connaissances des mutations à l'œuvre au sein des ICM. Elle permettrait par là de mieux comprendre les implications socio-économiques de l'achèvement du procès de numérisation des contenus pour l'ensemble du secteur. Enfin, nous verrons que ce type de recherche est en mesure d'éclairer le rôle grandissant joué par les industries de l'informatique et des télécommunications dans la distribution et la promotion des contenus culturels numérisés (Bouquillion, 2008; Bouquillion \& Matthews, 2010; Chantepie \& Le Diberder, 2010).

\section{UN LOISIR ET UN SECTEUR ECONOMIQUE EN VOIE DE LEGITIMATION ARTISTIQUE}

Au début des années 90 , après la crise qu'a connue le secteur dans les années 80 et qui s'est traduite par la fermeture de nombreuses entreprises et une baisse significative des revenus (Blanchet, 2010), l'industrie du jeu vidéo se présente plus que jamais comme une industrie culturelle en voie d'affirmation. A l'époque, la croissance de l'activité est principalement soutenue par les sorties successives des consoles Megadrive et Super Nintendo, la concurrence entre ces deux plateformes contribuant au dynamisme du secteur dans son ensemble. Dans le même temps, face à ce qui est en train de devenir un loisir de masse et une pratique culturelle en tant que telle pour les publics adolescents, de nombreux acteurs politiques, associatifs et même académiques tiennent des discours qui s'inscrivent pour la plupart dans le registre de la dénonciation des dangers supposés des jeux vidéo (Le Diberder, 1993). Les frères Le Diberder soulignent à cet effet qu'une grande partie d'entre eux peuvent aisément être mis en parallèle avec ceux qui accompagnèrent l'émergence du cinéma dans les années 20. Ces auteurs citent notamment Georges Duhamel qui qualifiait à l'époque le cinéma de "passe-temps d'illettrés, de créatures misérables, ahuries par leur besogne, une 
machine d'abêtissement et de dissolution» (ibid., p. 139). Si les termes choisis pour qualifier la pratique du jeu vidéo ont pu différer - en tentant notamment de placer les arguments avancés dans le registre de la scientificité - le fond des critiques était sensiblement le même. En substance, ces dernières amenaient à présenter le jeu vidéo comme une activité fondamentalement abrutissante, génératrice de comportements potentiellement violents et, en dernière analyse, susceptible de venir dissoudre toutes formes de lien social (Kline \& alii, 2003 ; Amato \& Perenny, 2008 ; Genvo, 2006, 2009). Nous pouvons supposer au passage que nous avons là une des explications du peu d'entrain des chercheurs travaillant sur les mutations des ICM pour inclure cet objet dans leurs réflexions théoriques.

Pour des raisons relevant tout autant de l'importance économique, culturelle et sociale prise par le jeu vidéo, que des actions de lobbying menées par les acteurs de la filière auprès des pouvoirs publics, force est de constater que la teneur des discours a aujourd'hui profondément changé. Que l'on place la focale au niveau politique, économique ou réglementaire, il est indéniable que le jeu vidéo est à présent considéré comme un bien culturel légitime à part entière (Natkin, 2004; Genvo, 2006, 2009), c'est-à-dire comme " une forme d'expression originale, capable de produire autant de chefs d'œuvre que de navets» (Natkin, 2004, p. 22). Entre autres événements significatifs, la mise en place depuis septembre 2009 d'un Observatoire du jeu vidéo piloté par le Centre national du cinéma et de l'image animée (CNC) vient concrétiser en France un long processus de reconnaissance du jeu vidéo par les autorités de tutelle. La naissance de cet observatoire signifie en substance que la création vidéoludique, du point de vue des instances de légitimation étatique, serait à mettre au même niveau que le cinéma, la musique ou l'édition. A ce titre, et ce n'est pas là l'un des moindres enjeux, cette reconnaissance permet aux acteurs de la filière de pouvoir prétendre à toute une série d'aides, sous forme notamment de crédits d'impôt prévus pour soutenir la création en France (Genvo, 2006, 2009 ; Blanchet, 2010). On peut voir ces différentes évolutions comme un aboutissement de l'intérêt porté, depuis une dizaine d'années, par les pouvoirs publics à un secteur économique où quelques acteurs nationaux occupent une place importante.

Car parmi les dix premiers éditeurs dans le monde en termes de chiffre d'affaires, deux voient leur capital majoritairement détenu par des firmes françaises. Il s'agit tout d'abord d'Activision Blizzard dont Vivendi est actionnaire à 61\%. Ce groupe affichait en 2010 un chiffre d'affaires de plus de trois milliards d'euros et reste l'actuel leader du marché mondial ${ }^{2}$. Le second éditeur français de dimension internationale est Ubisoft, cette entreprise ayant dégagé un chiffre d'affaires de 871 millions d'euros sur l'exercice $2010^{3}$. De plus, il convient de relever que le secteur du jeu vidéo représente «la seule industrie culturelle où les entreprises françaises exportent plus des trois quarts de leur production, éditant régulièrement des succès internationaux " (Le Diberder, 2002, p. 2). La France a donc de réels intérêts économiques à faire valoir dans le développement du marché vidéoludique, intérêts qu'elle entend manifestement protéger. Au surplus, si on analyse le marché du jeu vidéo français (hors consoles) on remarque que sur les 1,5 milliard d'euros qu'il a générés en 2009, les jeux édités par des entreprises françaises représentaient de l'ordre de $7 \%$ du volume total des ventes ${ }^{4}$. Bien que les chiffres soient légèrement en recul par rapport à 2008, le pourcentage reste néanmoins significatif.

\footnotetext{
${ }^{2}$ Source : Vivendi, « Rapport annuel - Document de référence 2010 ».

${ }^{3}$ Source : Ubisoft, « Document de référence $2010 »$.

${ }^{4}$ Source : Centre National du Cinéma et de l'image animée (CNC), « Le marché du jeu vidéo en 2009 ».
} 
Nous voyons donc que la reconnaissance artistique du jeu vidéo est ici profondément reliée à des considérations d'ordre économique et industriel. En effet, l'industrie vidéoludique revêt de plus en plus un caractère stratégique pour les pouvoirs publics. La création d'un Observatoire du jeu vidéo répond de ce fait à deux objectifs indissociables l'un de l'autre. En premier lieu, il s'agit d'accompagner, voire d'encadrer, un processus de légitimation artistique du jeu vidéo. Par ailleurs, les organismes de tutelle entendent mieux connaître un secteur économique sur lequel des entreprises françaises ont des intérêts à défendre. Sur ce point, le ministre de la Culture Frédéric Mitterrand a rappelé, au moment du lancement de l'observatoire, que l'un de ses principaux objectifs était « [d'aboutir] à des solutions claires afin de renforcer la condition de la création, sécuriser les investissements et favoriser la solidité financière des entreprises $»^{5}$. La reconnaissance du jeu vidéo en tant que « $10^{\text {ème }}$ art » est donc aussi un moyen d'aboutir à une meilleure définition de son statut juridique. Car celui-ci navigue, de manière souvent un peu floue, entre droits de propriété du logiciel de l'éditeur et un droit d'auteur en voie de constitution (Chantepie \& Le Diberder, 2010). De plus, nous avons aussi souligné qu'à partir du moment où le jeu vidéo parvient à accéder pleinement au rang d'œuvre de l'esprit, il peut dès lors prétendre bénéficier des dispositions réglementaires spécifiques qui en découlent. Au-delà des enjeux proprement esthétiques, la reconnaissance artistique est donc aussi un moyen d'assurer la protection juridique des jeux vidéo, principalement face aux risques d'appropriations illégales des contenus rendues possibles par Internet et le numérique.

En France, cette reconnaissance artistique doit tout autant aux actions menées par les organismes de tutelle qu'à celles, plus informelles mais parfois très organisées, des amateurs de jeux vidéo eux-mêmes. Du côté des pouvoirs publics, au-delà de la création de l'observatoire susmentionné, la mise en place en octobre 2005 d'un crédit d'impôt à destination des acteurs du secteur participe d'une démarche plus globale visant la pleine reconnaissance du jeu vidéo comme une forme de création culturelle et artistique originale. Mais les amateurs jouent eux aussi un rôle important notamment au niveau du processus de patrimonialisation du jeu vidéo en contribuant activement à la constitution d'une mémoire de cette jeune industrie. Ainsi, ce sont majoritairement eux qui facilitent l'accès, le téléchargement et l'émulation de jeux dits abandonware, c'est-à-dire de jeux qui ne sont plus commercialisés (Genvo, 2006, 2009). L'émulation consiste à rendre jouables sur son ordinateur personnel des titres initialement prévus et développés pour d'autres types de plateformes. Nous sommes dans ce cas à la limite de la légalité car « la délimitation de ce qui peut être ou non considéré comme abandonware est tout à fait arbitraire, la noncommercialisation d'un logiciel ne signifiant pas automatiquement son abandon par les détenteurs actuels des droits du jeu»(Genvo, 2006, p. 103). Cette tendance est donc d'un certain point de vue très profitable aux éditeurs car elle participe d'une reconnaissance artistique du jeu vidéo dans son ensemble (reconnaissance donnant droit, comme nous venons de le voir, à toute une série d'aides financières). Mais elle pose néanmoins problème dans la perspective d'une exploitation commerciale de leurs fonds de catalogue sur les nouveaux terminaux numériques tels que les smartphones ou plus récemment les tablettes.

Dans ce contexte, à la question de savoir si le jeu vidéo peut être considéré comme un bien culturel à part entière, Genvo et Solinski n'hésitent donc pas à répondre positivement

\footnotetext{
${ }^{5}$ Source : LePoint.fr, «Frédéric Mitterrand annonce la création d'un observatoire », URL :

http:/ / www.lepoint.fr/actualites-technologie-internet/2009-09-18/jeux-video-frederic-mitterrand-annonce-lacreation-d-un-observatoire/1387/0/378553 (consulté le 15/03/2011).
} 
(Genvo \& Solinski, 2010). Ces auteurs mobilisent notamment les travaux de Warnier pour justifier ce postulat, considérant que l'activité principale des acteurs de l'industrie vidéoludique consiste effectivement à " [produire] et [commercialiser] des discours, sons, images, arts" (ibid.). Si nous souscrivons à leurs conclusions, nous considérons néanmoins que le travail de justification théorique doit être complété avec d'autres recherches relevant plus spécifiquement des théories des mutations des ICM. Nous reviendrons plus loin sur un des points soulevés par Genvo et Solinski, à savoir la faible durée de vie commerciale des titres mis sur le marché. Sans qu'ils présentent cette spécificité comme étant le seul ni le principal frein à la reconnaissance du jeu vidéo comme un bien culturel (l'absence de construction institutionnalisée d'une mémoire collective du jeu vidéo leur semblant plus déterminante sur ce point), les auteurs considèrent néanmoins que cela a pu ralentir la progression du processus de légitimation artistique. A l'inverse, nous verrons dans la suite de notre argumentation que, pris dans une autre perspective théorique, nous avons là un trait caractéristique du fonctionnement socio-économique d'un marché des biens culturels. Dès lors, le jeu vidéo doit être considéré comme l'un d'entre eux, au même titre que le livre, le disque ou le DVD vidéo.

\section{LA THEORIE DES INDUSTRIES CULTURELLES COMME GRILLE D'ANALYSE DE LA FILIERE DU JEU VIDEO...}

Pour appuyer notre raisonnement, nous devons donc revenir sur les principaux apports de recherches entamées au début des années 70 et dont Capitalisme et industries culturelles fut l'un des premiers ouvrages à rendre compte de manière systématique (Huet \& alii, 1984). De nombreux travaux suivirent par la suite et vinrent compléter cette première publication. Certains auteurs (Miège, 2000; Moglin, 2007 ; Tremblay, 2008) ont tenté, dans une période récente, d'en faire une synthèse sur laquelle nous allons maintenant revenir. En nous appuyant sur les principaux apports de ces travaux, l'objectif est pour nous de proposer une assise théorique nous permettant d'envisager le secteur du jeu vidéo en tant que filière des industries culturelles.

En premier lieu, Tremblay rappelle à juste titre que «les chercheurs (...) s'entendent généralement pour reconnaître que les industries culturelles présentent des caractéristiques qui, prises une à une, peuvent se rencontrer dans d'autres secteurs industriels, mais qui, prises dans leur ensemble, ne se retrouvent que dans ce secteur auquel elles configurent un profil particulier» (Tremblay, 2008, p. 70). C'est donc bien tout un ensemble de spécificités qu'il convient de prendre en considération afin d'être en mesure de qualifier un secteur comme relevant des ICM. De ce fait, nous allons donc revenir sur ces différentes caractéristiques afin de montrer que l'industrie du jeu vidéo y correspond finalement point par point.

Tout d'abord, les industries culturelles doivent être appréhendées comme des industries de prototype. Cela signifie que chaque nouveau titre est un nouveau projet en tant que tel et nécessite la mobilisation en nombre de personnels très qualifiés et issus de secteurs professionnels dits créatifs. En d'autres termes, la phase de production d'un nouveau bien reste très largement artisanale et donc peu «standardisable ». Ici c'est bien l'importance du travail collectif de création qu'il faut souligner et, malgré de multiples tentatives allant dans ce sens, celui-ci reste difficilement mécanisable. Il y a ainsi une dimension profondément collective dans le processus de production/création, dimension que l'on retrouve également au moment de la conception d'un nouveau titre vidéoludique. Nous constatons ainsi que cette phase nécessite l'intervention de programmateurs, de graphistes, de concepteurs sonores mais aussi de scénaristes et de professionnels de la narration (Natkin, 2004). Tout comme pour le cinéma, l'édition ou le secteur phonographique, la filière du 
jeu vidéo est donc elle aussi artisanale dans sa phase de conception et industrielle dans celle de sa reproduction. Dans tous les cas, nous avons affaire à une industrie dont les coûts fixes sont très élevés et surtout en grande partie irrécupérables pour l'éditeur (Møglin, 2007). Le jeu vidéo s'inscrit très largement dans cette tendance puisque certains titres sortis ces dernières années ont des coûts de production s'élevant parfois jusqu'à 100 millions d'euros (Blanchet, 2010 ; Genvo \& Solinski, 2010), sans que le succès soit garanti pour autant.

Ceci nous amène sur une autre dimension structurante des ICM qui est celle du caractère fondamentalement aléatoire des valeurs d'usage des biens culturels industrialisés mis sur le marché. Les biens culturels (qu'ils soient industrialisés ou non) sont des biens dits d'expérience, ce qui signifie que le consommateur peut difficilement savoir, avant d'en avoir fait l'usage, si le contenu qu'il s'est procuré est susceptible de répondre à ses attentes esthétiques, ludiques, artistiques, informatives, etc. Ceci implique que le succès de tel ou tel titre ne pourra effectivement se mesurer qu'au moment de sa commercialisation. Comme le souligne Mœglin, "l'incertitude est omniprésente dans les industries et les marchés culturels. Elle affecte chaque produit nouveau - livre, film, jeu vidéo, etc. - lors de sa mise sur le marché. Désireux d'anticiper, stimuler, encadrer une demande peu prévisible et encore moins maîtrisable, les industriels rencontrent à chaque fois de semblables obstacles. (...) Les best-sellers se programment difficilement, quoi que l'on en dise après coup » (Moglin, 2007, p. 152). Le jeu vidéo n'échappe pas à cette constante de l'incertitude qui, loin d'être un signe de manque de maturité du marché, est au contraire révélateur de son appartenance pleine et entière au secteur des ICM. Nous pouvons d'ailleurs remarquer que, tout comme pour le cinéma, les acteurs de la filière vidéoludique n'hésitent pas à recourir à des stratégies de sérialisation en rééditant des formules éprouvées, voire tout simplement à adapter des films à succès (Blanchet, 2010). Ce point peut facilement être illustré puisqu'on remarquera que des titres tels que Guitar Hero, Street Fighter ou Les Sims ont déjà connu de nombreuses suites et ont donné lieu à un nombre encore plus grand d'imitations.

Une autre stratégie mise en place par les acteurs de l'industrie pour tenter de faire face à cette incertitude est celle du renouvellement permanent des titres commercialisés. L'obsolescence rapide des biens culturels doit donc être vue comme une caractéristique structurante propre à ce marché. Les éditeurs se voient donc dans l'obligation de proposer constamment de nouveaux titres pour répondre à une demande des plus versatiles. L'idée générale qui sous-tend cette observation est qu'au final les quelques succès commerciaux (qui relèvent de l'exception dans le cadre des ICM) permettent de financer l'intégralité du catalogue dont la très grande majorité des titres n'aura généralement pas réussi à amortir ses coûts de production. Cette dialectique dite du tube et du catalogue (Miège, 2000, 2007 ; Mœglin, 2007) doit donc être prise pour ce qu'elle est, à savoir une stratégie industrielle devant permettre l'équilibre économique de l'ensemble de la filière. Dès lors, le point relevé par Genvo et Solinski ainsi que par d'autres auteurs (Daidj \& Isckia, 2009 ; Blanchet, 2010) concernant la très forte rotation des titres de jeux vidéo mis sur le marché ne doit pas être perçu comme un obstacle théorique à leur inclusion dans le secteur des ICM, mais bien au contraire comme une des marques de leur pleine appartenance à celui-ci. Blanchet souligne à ce propos que pendant la période précédant les fêtes de fin d'année, il peut sortir entre 15 à 20 nouveautés par semaine (Blanchet, 2010).

Ces évolutions ont eu pour principales conséquences d'entraîner une très forte inflation des coûts de promotion et un net mouvement de polarisation entre contenus premium et marchés de niche. Quant aux stratégies de valorisation marchande du catalogue ainsi constitué, il semblerait que les acteurs de la filière commencent tout juste à mettre en 
œuvre des offres allant dans ce sens. Elles ont été notamment rendues possibles grâce aux consoles de dernière génération telles que la Xbox 360 et la Playstation 3. Chacune d'entre elles permet de se connecter à une plateforme dédiée via Internet (Xbox Live et Playstation Network) et de télécharger, moyennant paiement en ligne, d'anciens titres ayant connu un succès commercial sur les consoles des générations précédentes.

Une autre conséquence de cette incertitude sur les valeurs d'usage est un déplacement de la prise en charge du risque éditorial et financier vers l'amont de la filière, là où se situent généralement les structures les plus fragiles. Dans le cas du jeu vidéo, il existe une articulation particulière entre les trois types d'acteurs que l'on retrouve dans les autres filières des ICM. Il y a tout d'abord les éditeurs qui prennent en charge les fonctions qui sont dévolues au producteur tel qu'il est défini dans le cadre du modèle éditorial (Miège, 2000). Leur rôle consiste à avancer l'argent couvrant tout ou partie des coûts de production, assurer les fonctions marketing et orienter le choix des projets qui seront mis en place et commercialisés. D'une certaine manière, les éditeurs jouent le rôle d'interface entre la dimension créative du projet et le marché.

En amont de la filière, on retrouve le second type d'acteurs que sont les studios de développement vidéoludique. Soit ces derniers proposent des projets qu'ils ont eux-mêmes produits à des éditeurs, soit ils tentent de répondre à un appel d'offre des éditeurs (Kline \& alii, 2003 ; Natkin, 2004). En toute rigueur, ce sont bien les studios qui subissent la plus grosse part du risque dans la mesure où un projet lancé par un éditeur peut très bien être stoppé en cours de développement, menaçant ainsi leur existence même. Là aussi, le report du risque généré par l'incertitude vers l'amont de la filière - où l'on retrouve des acteurs de petite taille et plus fragiles économiquement - est une constante observée dans le secteur du jeu vidéo comme dans toutes les filières des ICM à des degrés divers. Il en va de même concernant une tendance à la concentration du secteur de l'édition qui est là aussi un moyen de lutter contre l'incertitude en jouant sur la taille et donc sur la capacité de financement de projets toujours plus coûteux. Kline et alii soulignent à cet égard que la filière du jeu vidéo, dans sa structuration même, a finalement de nombreux points communs avec celle de la musique enregistrée (Kline \& alii, 2003). Ces auteurs soulignent ainsi que les studios de développement peuvent être vus comme des groupes de musique qui seront ensuite signés par un éditeur, ce dernier jouant le rôle dévolu à la maison de disque dans l'industrie phonographique.

Le dernier type d'acteurs, sur lequel nous reviendrons dans notre troisième partie, est celui des fabricants de consoles de jeux. Actuellement, $60 \%$ du chiffre d'affaires généré sur le marché vidéoludique provient de celui des consoles, ce qui contribue à renforcer la position des trois fabricants qui dominent seuls ce secteur (Sony, Microsoft et Nintendo) (Kline \& alii, 2003 ; Natkin, 2004). Aucune des plateformes développées par ces derniers ne sont interopérables entre elles. De ce fait, nous nous retrouvons dans une sorte de «faux oligopole " puisqu'en réalité chacune de ces firmes s'est constituée son propre monopole dans lequel le consommateur se retrouve très largement captif (Chantepie \& Le Diberder, 2010). Par ailleurs, ce sont les fabricants (ou consoliers) qui valident ou non la commercialisation de tel ou tel titre, en assurent la distribution, imposent un cahier des charges précis à destination des éditeurs et se réservent de l'ordre de $22 \%$ du prix de vente (contre $29 \%$ pour l'éditeur, $14 \%$ pour le développeur et $35 \%$ pour le détaillant) (Natkin, 2004). Les fabricants de consoles agissent donc comme un véritable goulot d'étranglement et comme des intermédiaires incontournables pour accéder à la demande. De plus, les consoliers peuvent aussi prendre en charge la partie édition, faisant dès lors passer la part qui leur revient à plus de 50\%. Nous voyons là une illustration très marquée d'une 
domination de l'aval sur l'amont de la filière constatable dans les industries culturelles dans leur globalité mais selon des modalités propres au jeu vidéo.

Les différents points que nous venons de repérer nous semblent démontrer que l'inclusion des jeux vidéo dans le secteur des ICM est théoriquement fondée. Partant de ce postulat, nous devons maintenant opérer le chemin inverse. Dans une troisième partie, nous entendons donc exposer en quoi l'étude des spécificités de l'industrie vidéoludique est susceptible d'enrichir la compréhension des mutations en cours au sein des industries culturelles prises dans leur ensemble. Par ailleurs, nous verrons pourquoi une compréhension des logiques de fonctionnement socio-économique de l'industrie vidéoludique permet d'éclairer l'évolution des relations des industries des contenus avec celles de l'informatique et les opérateurs des télécommunications. A cette fin, nous entendons-nous placer aussi bien dans le cadre du procès de constitution d'un secteur plus englobant qui serait celui des industries de la culture, de l'information et de la communication (ICIC) (Miège, 2000), que dans celui de l'analyse des mouvements de " coordination tendancielle » entre industries des contenus, des réseaux et des matériels (Miège, 2004).

\section{... L'ETUDE DE LA FILIERE DU JEU VIDEO POUR ENRICHIR LA THEORIE DES INDUSTRIES CULTURELLES}

Au même titre que le cinéma, le jeu vidéo doit être considéré comme une nouvelle forme de création qui n'a pu émerger que dans le cadre du procès d'industrialisation entamé aux XVIII $^{\text {ème }}$ et XIX ${ }^{\text {ème }}$ siècles. Miège présente ainsi le cinéma comme «le produit culturel industrialisé le plus complet et celui qui, assurément, anticipe sur des développements futurs » (Miège, 2000, p. 47-48). L'auteur considère également que si le cinéma doit être appréhendé comme le premier art industriel c'est avant tout parce que celui-ci « à partir d’une copie "zéro", réalisée par une équipe artistique et technique, placée sous la responsabilité (...) d'un producteur, et du tirage qui est fait des copies pour la diffusion, celle-ci se déroule (...) sans qu'il soit besoin de faire appel (...) aux artistes interprètes et aux techniciens» (ibid., p. 48). De ce point de vue, le cinéma est donc le premier art industriel au sens où les phases de production et de reproduction sont radicalement séparées. Dans une large mesure, ses codes narratifs se sont constitués autour de cette spécificité. Il n'en va pas exactement de même pour la musique, par exemple. Dans ce cas, nous avons affaire à un mode d'expression artistique qui préexistait à l'invention du phonographe par Edison et qui s'est finalement retrouvé dans une situation qui était plus celle d'un «faire avec» les techniques de reproduction industrielles. Si cette innovation technique a participé de l'émergence d'une pratique culturelle nouvelle autour de l'écoute privatisée de la musique - et d'un marché de type capitaliste rendu dès lors possible par les techniques de fixation et de reproduction du son - elle n'a pas, dans un premier temps, donné naissance à un type de création radicalement nouveau (Perticoz, 2009). Dans ce cas précis, si la technique a par la suite favorisé une évolution des modes d'expressions musicaux, elle a surtout très largement bousculé les modalités de valorisation marchande qui prédominaient jusque-là. Sur ce dernier point, c'est bien en étudiant les spécificités du cinéma qu'il a été possible d'en comprendre les logiques et d'être ainsi en mesure de mieux les appréhender.

Plus d'un siècle plus tard, nous pensons que des parallèles peuvent être dressés avec le jeu vidéo. Comme le cinéma, il s'agit d'une forme d'expression artistique et culturelle qui ne peut s'envisager hors du procès d'industrialisation. Il nous semble également que la création vidéoludique donne à voir une toute autre dimension des mutations en cours au sein des ICM. Plus précisément, l'étude de la filière jeu vidéo offre un terrain d'observation 
privilégié des implications socio-économiques induites par l'achèvement du procès de numérisation des contenus culturels et informationnels. Car si le cinéma est le premier art industriel en tant que tel, le jeu vidéo peut être considéré pour sa part comme le premier art numérique (Chantepie \& Le Diberder, 2010), d'autres auteurs allant même jusqu'à parler de premier cybermédium (Amato \& Perenny, 2008). Dans cette perspective, Amato et Perenny considèrent ainsi que «le jeu vidéo apparaît comme le vecteur de l'humanisation et de la promotion de l'informatique populaire de masse » (ibid., p. 9). Cette proposition mérite d'être discutée car elle est susceptible de venir enrichir les recherches sur les relations entre les industries de contenus et celles de la communication et de l'informatique. Car sans l'informatique et la numérisation - en tant que conditions nécessaires mais non suffisantes - l'avènement du jeu vidéo n'est effectivement pas envisageable. Par ailleurs, les jeux vidéo représentent bel et bien un des types de contenus les plus prisés sur les nouveaux terminaux numériques tels que l'iPad. Au-delà d'une indéniable " promotion de l'informatique populaire de masse ", les jeux vidéo favorisent la vente - et justifient l'achat - de matériels électroniques grand public commercialisés principalement par l'industrie informatique.

De plus, le cinéma, la musique enregistrée ou l'édition de livres ont été - ou sont encore dans une démarche qui ressemblait davantage à une tentative d'intégration de la numérisation dans leurs modes de production/valorisation, celle-ci leur était en quelque sorte extérieure. Ces industries ont, selon les cas, essayé d'en tirer le meilleur parti (effets spéciaux numériques, abaissement des coûts de production, meilleures diffusion des œuvres, etc.) ou de "faire avec" (majoritairement sur les questions de droit d'auteur dans l'environnement numérique et du " piratage » qui s'en trouve dès lors facilité). Concernant le jeu vidéo, la numérisation est d'une certaine manière inscrite dans son mode de production même. Par ailleurs, si au niveau de l'aval de la chaîne de production/valorisation de la création vidéoludique le numérique n’a commencé que très récemment à jouer un rôle, celle-ci fut néanmoins parmi les premiers biens culturels à utiliser les réseaux numériques dans sa phase de commercialisation. Face à l'achèvement du procès de numérisation, l'étude de cette filière et de ses particularités semble donc en mesure d'offrir de nombreux éclairages quant aux mutations observables actuellement au sein des ICM.

Le jeu vidéo est le premier - et pour l'instant le seul - type de création original qui intègre pleinement les spécificités du multimédia, tant au niveau de la conception que de celui des différentes formes de pratiques culturelles qu'il génère (Miège, 2007). En dernière analyse, des recherches approfondies sur cette filière devraient nous permettre " (d'anticiper) sur des développements futurs » de l'ensemble du secteur, que ce soit au niveau de modes de production, de distribution ou de valorisation des contenus.

Nous voyons ainsi se dégager plusieurs tendances dans le secteur du jeu vidéo qui s'observent aussi à des degrés divers au sein des autres filières des ICM. Il s'agit tout d'abord d'un renforcement de la domination de l'aval et de la place de plus en plus grande prise par les terminaux numériques en tous genres. Nous avons pu remarquer dans le cas du marché du jeu vidéo, et plus précisément celui des consoles, que le terminal joue un rôle prépondérant et conditionne toute la structuration de la filière en amont. Les éditeurs - et encore plus les studios de développement - doivent composer avec les consoliers qui représentent l'un de leurs principaux débouchés économiques. Ceci ne signifie néanmoins pas que ces mêmes éditeurs seraient complètement soumis aux logiques industrielles des fabricants de consoles. En effet, ces derniers doivent aussi s'assurer qu'un nombre suffisant de titres premium seront présents au lancement d'un nouveau modèle, ne serait-ce que pour garantir un niveau élevé de ventes. On parle parfois dans ce cas d'un modèle nommé 
«razor and blades» où les fabricants vendent leur console à des prix relativement bas afin qu'un maximum de foyers décide de s'équiper (Daidj \& Isckia, 2009). L’idée ici est de se garantir des revenus substantiels grâce à la vente de titres, considérant qu'à partir du moment où les amateurs de jeux vidéo ont investi dans tel ou tel type de plateforme, ils se trouvent ensuite dans une posture relativement captive vis-à-vis du consolier et de son catalogue.

Par ailleurs, la dernière génération de consoles offre maintenant la possibilité de se connecter à Internet, ces dernières pouvant dès lors prétendre au statut de terminaux de loisirs numériques en tous genres. Les Xbox 360 et autres Playstation 3 permettent d'accéder à des services de vidéo à la demande ou de téléchargement de musique en ligne. Dans cette optique, on peut envisager des modalités croisées de valorisation marchande où l'achat d'un jeu vidéo pourrait ensuite donner lieu au téléchargement d'un film. C'est inscrit dans cette perspective économique que le contrôle du terminal est considéré comme éminemment stratégique par les industriels du secteur. Entre autres enjeux, ce type de contrôle doit permettre d'être en prise directe avec le consommateur final. L'objectif et d'être ainsi en mesure de lui proposer toute une gamme de contenus culturels et de services diversifiés et d'être l'opérateur qui contrôlera sa facture. C'est à ce titre que l'étude de la filière du jeu vidéo peut aussi nous aider à mieux comprendre les « relations qui s'organisent entre industries de réseaux, industries de matériels et industries de programmes » (Miège, 2007, p. 31).

Car les jeux vidéo arrivent de plus en plus comme un simple complément - certes à forte valeur ajoutée - des offres d'abonnement des opérateurs de télécommunication. La dernière Freebox mise sur le marché par Iliad est ainsi proposée avec une manette en plus de la télécommande classique. L'éditeur Gameloft offre déjà la possibilité de télécharger cinq titres sur la plateforme numérique Freestore pour moins de dix euros (contre cinquante euros en moyenne pour les titres édités sur consoles). Du côté du marché des tablettes numériques dominé par Apple ou de celui des smartphones, les jeux vidéo représentent là aussi l'un des contenus les plus prisés. Ainsi, en 2010, les jeux vidéo représentaient $25 \%$ des applications téléchargées sur l'iPhone.

Ces évolutions récentes, en plus de la potentielle remise en cause de la domination des consoliers au niveau de l'aval, sont de nature à confirmer l'importance prise par les opérateurs de télécommunications et l'industrie informatique dans la distribution de contenus culturels. Avec le recul, nous voyons que la manière dont la filière des jeux vidéo s'est structurée dès ses débuts préfigurait, en quelque sorte, la perte de contrôle sur la distribution de leurs contenus que connaissent actuellement les acteurs des industries de la musique enregistrée, de l'édition et, dans une moindre mesure, du cinéma. Comme nous l'avons vu, même les éditeurs de jeux vidéo les plus importants ont toujours dû composer avec des fabricants de matériels qui faisaient office de goulot d'étranglement au niveau de la distribution. C'est cette situation, relativement nouvelle pour les acteurs de ces filières " traditionnelles », que sont en train d'expérimenter les majors du disque, du cinéma et de l'édition avec Apple, Google ou Free. Ces derniers n'interviennent pas, ou alors de manière très marginale, dans la phase de production et se contentent d'offrir un moyen de diffusion sur les réseaux numériques en prélevant un pourcentage du prix de vente ou en se rémunérant avec les revenus publicitaires. Ces évolutions doivent donc aussi nous

\footnotetext{
${ }^{6}$ Source : JournalduNet.com, «Une application de l'App Store sur quatre est un jeu », URL : http://www.journaldunet.com/ebusiness/telecoms-fai/applications-app-store-france/repartition-parcategories.shtml (consulté le 28/03/2011).
} 
interroger sur la pertinence des modèles génériques que sont le modèle éditorial, le modèle de flot et leurs déclinaisons (Miège, 2000 ; Mœglin, 2007) dans le cadre du passage au numérique, l'étude de la filière jeu vidéo devant permettre de nous éclairer sur ce point.

Par ailleurs, c'est bien au niveau des stratégies dites multi-supports (Bouquillion, 2008) que des recherches approfondies sur la filière du jeu vidéo seraient susceptibles d'offrir des enseignements précieux. Si les jeux sur consoles continuent, comme nous l'avons vu, à dominer très largement le secteur, les jeux pour ordinateurs personnels ainsi que ceux développés pour les nouveaux terminaux mobiles se partagent respectivement $21 \%$ et $19 \%$ du marché (Genvo \& Solinski, 2010). Dans le cas des jeux sur terminaux dits nomades, les consoles de jeux portables telles la PSP de Sony ou la DS de Nintendo captent encore la majeure partie du chiffre d'affaires généré. Néanmoins, il nous faut rappeler que la popularité grandissante des produits d'Apple tels que l'iPhone et plus récemment l'iPad semblait en mesure de remettre en cause certaines positions acquises. Nombre d'acteurs de la filière voient notamment dans l'arrivée des tablettes tactiles un moyen de faire émerger de nouvelles places de marché à même d'assurer l'articulation entre le secteur de la téléphonie et des consoles portables (ibid.). Mais il apparaît de plus en plus que cette émergence se fait aux conditions imposées par ces nouveaux acteurs de la distribution de contenus culturels numérisés. Au niveau de la répartition des revenus, on sait par exemple qu'Apple récupère $30 \%$ du prix de vente de chaque application payante téléchargée sur son App Store ${ }^{7}$. Une fois encore, même si les acteurs peuvent être amenés à changer, il reste que c'est bien l'effectivité d'un renforcement de la domination de l'aval qui semble se confirmer.

Nous pouvons néanmoins constater que les éditeurs sont aussi dans une dynamique de diversification de leurs sources de revenus afin d'être moins dépendants des consoliers en termes de débouchés. Les tablettes et les smartphones sont vus à cet égard comme de nouveaux terminaux aux perspectives prometteuses. D'un autre côté, les fabricants de ces terminaux mobiles voient dans les applications intégrant des jeux vidéo un moyen d'enrichir leurs offres de contenus disponibles et ainsi favoriser les ventes de leurs produits. Comme le souligne Miège, « la question des programmes est essentielle à la progression de l'ensemble du secteur » (Miège, 2007, p. 48), ce qui signifie notamment que les industries du matériel informatique, mais aussi celles des télécommunications, se doivent de porter une attention toute particulière à l'offre de contenus en tous genres. Sur ce point, le jeu vidéo est souvent considéré comme une filière possédant de réelles perspectives de croissance susceptible d'entraîner dans son sillage l'ensemble des acteurs du secteur des ICIC.

Nous pensons voir là l'illustration du renforcement des tendances à un déplacement vers les contenus et à l'affirmation d'une réciprocité des intérêts entre les producteurs de programmes, les fabricants de matériels et les opérateurs en télécommunication (Miège, 2007). Des entreprises telles qu'Apple ou Google ont tout d'abord pu avoir des stratégies assimilables à celle du "passager clandestin» (Bouquillion, 2008). Par exemple, Apple a dans un premier temps indirectement bénéficié du téléchargement illégal de musique enregistrée qui a pu stimuler les ventes de son iPod. Néanmoins, la tendance semble maintenant à la signature de partenariats et de contrats d'exploitation/distribution, afin d'assurer une progression des ventes de terminaux numériques en insistant sur la richesse de l'offre de contenus disponibles. Une fois encore, les conditions de ces accords

......

${ }^{7}$ Source : Apple.com, «Apple lance les abonnements sur l'App Store », URL :

http://www.apple.com/fr/pr/library/2011/02/15appstore.html (consulté le 28/03/2011). 
apparaissent dans une large mesure imposées par les acteurs de l'aval, ces derniers n'étant par ailleurs pas disposés à investir dans la production de contenus originaux (ibid.). Dès lors, face à cette domination de l'aval, seules les firmes en mesure de produire des contenus premium générateurs de fortes audiences gardent la possibilité d'infléchir les termes des contrats dans un sens qui leur serait plus favorable.

La reconduction récente de l'accord entre la société Facebook et Zynga, un éditeur de jeux vidéo dits de casual gaming, peut s'interpréter de cette manière. Les casual games sont des jeux aux règles simples, pouvant se jouer de manière occasionnelle et généralement intégrés aux navigateurs Internet sur les sites de réseaux sociaux. Considérant que les conditions financières imposées par Facebook pour héberger ses jeux n'étaient plus acceptables, Zynga a ainsi menacé de les retirer pour créer sa propre plateforme, l'obligeant finalement à lui proposer un contrat plus avantageux ${ }^{8}$. Les applications vidéoludiques de Zynga peuvent se prévaloir de près de 320 millions d'utilisateurs plus ou moins réguliers. Pour un site tel que Facebook, dont le principal objectif est de générer le plus de trafic possible sur sa plateforme, ce type de contenu est donc en passe de devenir des plus stratégiques. En d'autres termes, pour assurer la croissance de son activité, Facebook doit être capable de proposer des contenus attractifs, les jeux vidéo étant considérés comme les mieux placés. Cet épisode vient aussi illustrer le fait que les relations et les rapports de force entre les acteurs de l'amont et ceux de l'aval doivent être envisagés dans toute leur complexité. Comme le rappelle Catherine Vénica, «les synergies industrielles et les pouvoirs de marchés ne peuvent s'interpréter qu'en lien avec les éléments de connaissance plus spécifiques sur le fonctionnement socio-économique des industries de la culture et de la communication » (Vénica, 2007, p. 64).

De plus, ce déplacement vers les contenus, dont les jeux vidéo seraient l'un des fers de lance, n'est pas sans poser certaines questions quant aux logiques spéculatives qui seraient en train de se mettre en place autour de l'industrie vidéoludique. Les perspectives offertes par cette dernière sont vues comme étant particulièrement prometteuses. Nous avons déjà insisté sur le fait que nombre d'applications téléchargeables sur les smartphones sont des jeux vidéo. Les différents modèles de télévisions connectées (Google TV, Apple TV etc.) misent également beaucoup sur le téléchargement de jeux vidéo en ligne ${ }^{9}$. Dès lors, il est permis de se demander si nous ne serions pas en train de voir revenir des stratégies industrielles portées par des discours exagérément optimistes quant aux perspectives de croissance à court terme du secteur du jeu vidéo. A cet égard, ceux-ci ne sont pas sans rappeler ceux qui ont accompagné la formation de la «bulle Internet». Dans tous les cas, le fait que la capitalisation boursière d'une firme telle que Zynga (dont l'existence ne remonte qu'à 2007) ait pu s'élever à près de 5,51 milliards de dollars ${ }^{10}$ alors que les revenus générés par celle-ci sont loin d'être comparables à d'autres acteurs plus anciens de la filière, tels qu'Electronic Arts, doit nous interroger. Dans ce contexte, une approche de type socio-économique et les apports des travaux menés sur les fusions AOL/Time-Warner et Vivendi/Universal incitent à appréhender tous ces mouvements avec une indispensable prudence théorique (Bouquillion, 2007, 2008).

\footnotetext{
${ }^{8}$ Source : ElectronLibre.info, «Zynga et Facebook rabibochés pour 5 ans », URL : http://electronlibre.info/+Zynga-et-Facebook-rabiboches-pour,03141+ (consulté le 15/03/2011).

${ }^{9}$ Source : ElectronLibre.info, « Le casual gaming : la nouvelle bulle Internet », URL :

http://electronlibre.info/Le-casual-gaming-la-nouvelle-bulle,00968 (consulté le 15/03/2011).

${ }^{10}$ Source : GameCharts.fr, «Zynga dépasse Electronic Arts », URL : http://www.gamescharts.fr/2010/10/zyngadepasse-electronic-arts/ (consulté le 15/03/2011).
} 
Enfin, l'étude de la filière du jeu vidéo nous apparaît comme une entrée des plus heuristiques concernant les problématiques de recherche liées à l'idéologie sous-tendant les discours autour du web dit 2.0, notamment au niveau de l'émergence supposée « de la figure de l'amateur-créateur de contenus» (Bouquillion \& Matthews, 2010). Une mise en perspective des discours valorisant la capacité des consommateurs à être eux-mêmes producteurs de contenus, plus particulièrement dans le cadre des jeux en réseaux, relève d'une démarche théorique qu'il convient de systématiser. Bouquillion et Matthews soulignent qu'à l'aune d'une hypothétique montée en puissance de «l'économie affective », un idéal-type du consommateur serait en train de se dessiner où celui-ci serait " actif, émotionnellement engagé dans ses actes de consommation et intégré à des réseaux sociaux » (ibid., p. 63). Dans le cadre de cette proposition, il apparaît que des recherches autour des communautés de gamers, de par le rôle actif que certains semblent jouer dans l'amélioration du jeu, offrent des perspectives à approfondir. S'appuyant entre autres sur les travaux de Jenkins, Raessens souligne ainsi le fait que, bien plus que dans les filières traditionnelles, les acteurs de l'industrie vidéoludique intègrent l'activité des gamers dans leurs stratégies de production de nouveaux contenus (Raessens, 2005). L'auteur parle d'une culture du bottom-up où «les joueurs utilisent les technologies open-source et de partage de fichiers pour recycler les produits grand public des industries culturelles » (ibid., p. 382$)^{11}$.

Ce postulat serait nécessairement à nuancer car, au-delà de cette figure de l'usager créatif du côté duquel se déplacerait la création de valeur, c'est bien plutôt à une accentuation d'un mouvement de polarisation des contenus (Bouquillion, 2008) à laquelle, semble-t-il, nous sommes en train d'assister. Sur ce point, le secteur du jeu vidéo apparaît comme particulièrement à la pointe avec, d'un côté, des contenus premium conçus pour s'adresser au plus grand nombre et, de l'autre, une multitude de marchés de niches pour lesquels les consommateurs seraient mis à contribution. Concernant ce dernier, il conviendrait de préciser quelle est réellement la place de l'amateur de jeux vidéo dans le processus de création, notamment pour des applications au faible coût de production et dont le but premier est de générer du trafic sur les principaux réseaux sociaux. Sur les contenus premium, la détention par les éditeurs de jeux vidéo de licences au fort potentiel de notoriété apparaît de plus en plus incontournable et semble se substituer à la logique de star system qui prend des formes différentes au sein de cette filière. Les processus de création étant fondamentalement collectifs et assumés comme tels, l'enjeu se situerait plus dans la capacité des éditeurs à créer des personnages fédérateurs (Mario Bros, Tomb Raider, Solid Snake etc.) qui pourront ensuite avoir les honneurs d'une déclinaison cinématographique. D’un autre côté, les majors de la filière vidéoludique semblent résolument s'inscrire dans une politique d'achats de licences de contenus issus d'autres secteurs et pouvant donnant lieu à une adaptation vidéoludique (Harry Potter, Dragon Ball Z, les événements sportifs les plus médiatiques, etc.).

Au-delà de l'arrivée d'une nouvelle économie qui serait plus "affective ", l'étude critique de la filière du jeu vidéo doit donc nous amener à mettre au jour des tendances inscrites dans le temps long des mutations des ICM et, plus largement, des ICIC.

\footnotetext{
.......

11 « we encounter these bottom-up cultures where gamers use sharing and open-source techniques to recycle existing products of the mainstream practices of the cultural industries » (notre traduction).
} 


\section{CONCLUSION}

Au regard des différents points que nous avons développés, nous pensons donc que l'étude des spécificités socio-économiques de l'industrie des jeux vidéo ouvre d'indéniables perspectives théoriques. Nous considérons que cette démarche est susceptible de faire progresser significativement la recherche sur les mutations de l'ensemble des filières des ICM. Entre autres enjeux, il nous semble que la place qu'est en train de prendre le micropaiement au sein de l'industrie vidéoludique - dans le cadre des jeux en réseau ou du casual gamnig - doit nous inciter à interroger plus particulièrement la validité des modèles génériques et de leurs déclinaisons (Miège, 2000 ; Mœglin, 2007). Dès lors, en tant que premier bien culturel issu du procès de numérisation, le jeu vidéo représente à bien des égards un terrain qu'il convient de prendre pleinement en considération. Afin de préciser ces perspectives, nous entendons mener ces recherches dans le cadre d'une interrogation systématique des modalités d'articulation entre les évolutions des pratiques culturelles médiatisées par la technique et les mutations observables au sein des ICM/ICIC (Perticoz, 2009, 2010). Il s'agit pour nous d'être capable de mettre en relation les stratégies des acteurs industriels intervenant dans le secteur du jeu vidéo et la manière dont les amateurs vivent leur(s) pratique(s) de ce loisir au quotidien, l'intègrent dans leurs consommations et dans leurs modes de vie.

Dans cette optique, il apparaît alors nécessaire de convoquer la figure d'un individu supposé créatif et socialement valorisé dans le cadre d'un nouvel esprit du capitalisme (Boltanski \& Chiapello, 2011). Après examen, cet individu apparaît finalement et surtout comme un consommateur de contenus produits par les ICM (Bouquillion, 2007). Ce constat fait ainsi pleinement écho à la supposée instabilité de la demande des consommateurs qui serait elle-même le révélateur " des revendications propres à la critique artiste, notamment celles se rattachant à une exigence "d'authenticité », qui se sont traduites par la recherche de biens de consommation individualisant des consommateurs " ciblés », contrairement à toutes les productions «de masse » (Boltanski \& Chiapello, 2011, p. 931-932). Si on prend cette proposition au sérieux, c'est donc bien l'hypothèse de l'émergence d'une industrie des modes de vie ubiquitaires et présentés comme créatifs que des recherches sur la filière du jeu vidéo peuvent contribuer à mettre en lumière. 


\section{REFERENCES BIBLIOGRAPHIQUES}

Amato, Etienne Armand ; Perenny, Etienne (2008), « Comment le premier cybermédium a pu un temps échapper aux SIC? De la dynamique structurelle du jeu vidéo au Réseau », $X V I^{e}$ Congrès de la Société Française des Sciences de l'Information et de la Communication, Université Technologique de Compiègne [en ligne], http://www.omnsh.org/spip.php?article171, consulté le 15 mars 2011.

Boltanski, Luc; Chiapello, Ève (2011), Le nouvel esprit du capitalisme, Paris : Gallimard (collection «tel »).

Bouquillion, Philippe (2008), Les industries de la culture et de la communication. Les stratégies du capitalisme, Grenoble : PUG (collection «Communication médias société »).

Bouquillion, Philippe (2007), «Les industries de la culture, de l'information et de la communication dans le capitalisme ", in Bouquillion, Philippe ; Combès, Yolande (dir.), Les industries de la culture et de la communication en mutation, Paris : L'Harmattan.

Bouquillion, Philippe ; Matthews, Jacob (2010), Le web collaboratif. Mutations des industries de la culture et de la communication, Grenoble : PUG (collection « La communication en plus »).

Blanchet, Alexis (2010), Des pixels à Hollywood. Cinéma et jeu vidéo, une histoire économique et culturelle, Triel-sur-Seine : Editions Pix'n Love.

Chantepie, Philippe; Le Diberder, Alain (2010, $2^{\text {ème }}$ édition), Révolution numérique et industries culturelles, Paris : La Découverte (collection « Repères »).

Daidj, Nabyla ; Isckia, Thierry (2009), "Entering the Economic Models of Game Console Manufacturers" (p. 23-42), Communications $\mathcal{E}$ Strategies. DigiWorld Economic Journal, ${ }^{\circ} 73,1^{\text {st }}$ quarter 2009.

Dyer-Witheford, Nick; Peuter (de), Greig (2009), Global Capitalism and Video Games, Minneapolis : University of Minnesota Press.

Genvo, Sébastien (2009), Le jeu à son ère numérique. Comprendre et analyser les jeux vidéo, Paris : L'Harmattan (collection « Communication et Civilisation »).

Genvo, Sébastien (2006), Le game design de jeux vidéo: approche communicationnelle et interculturelle, thèse soutenue sous la direction de Jacques Walter, Metz: Université Paul Verlaine.

Genvo, Sébastien ; Solinski, Boris (2010), «Le jeu vidéo : un bien culturel ? », InaGlobal, la Revue des industries créatives et des médias [en ligne], http://www.inaglobal.fr/jeuvideo/article/le-jeu-video-un-bien-culturel?tq=8, consulté le 15 mars 2011.

Huet, Armel ; Ion, Jacques ; Lefèbvre, Alain ; Miège, Bernard ; Peron, René (1984, $2^{\text {ème }}$ édition), Capitalisme et industries culturelles, Grenoble : PUG (collection «Influences »).

Kline, Stephen; Dyer-Witheford, Nick; Peuter (de), Greig (2003), Digital Play. The Interaction of Technology, Culture and Marketing, Montréal : McGill-Queen's University Press.

Le Diberder, Alain; Le Diberder, Frédéric (1993), Qui a peur des jeux vidéo ?, Paris : La Découverte (collection « Essais »).

Le Diberder, Alain ; Le Diberder, Frédéric (2002), "La création de jeux vidéo en France en 2001 », Bulletin du département des études et de la prospective, 139 [en ligne], http://www2.culture.gouv.fr/culture/deps/2008/pdf/dc139.pdf, consulté le 15 mars 2011.

Miège, Bernard (2000), Les industries du contenu face à l'ordre informationnel, Grenoble : PUG (collection « La communication en plus »). 
Miège, Bernard (2004), L'information - communication, objet de connaissance, Bruxelles (Belgique) : De Boeck \& INA.

Miège, Bernard (2007, 2 $2^{\text {ème }}$ édition), La société conquise par la communication. 2. La communication entre l'industrie et l'espace public, Grenoble : PUG (collection « Communication médias société »).

Mœglin, Pierre (2007), «Des modèles socio-économiques en mutation» (p. 151-162), in Bouquillion, Philippe; Combès, Yolande (dir.), Les industries de la culture et de la communication en mutation, Paris : L'Harmattan (collection "Questions contemporaines »).

Natkin, Stéphane (2004), Jeux vidéo et médias du XXI' siècle. Quels modèles pour les nouveaux loisirs numériques? Paris : Vuibert.

Perticoz, Lucien (2010), «Musique au jour le jour, construction de soi et mutations de l'industrie musicale : une approche par le quotidien » (p. 70-79), Revista românã de Jurnalism si Comunicare, Anul V (VIII), nr. 2 (31).

Perticoz, Lucien (2009), Les processus techniques et les mutations de l'industrie musicale. L'auditeur au quotidien, une dynamique de changement, thèse soutenue sous la direction de Bernard Miège, Grenoble : Université Stendhal Grenoble 3.

Raessens, Joost (2005), "Computer games as participatory media culture » (p. 373-388), in Raessens, Joost; Goldstein, Jeffrey (dir.), Handbook of computer game studies, Cambridge (Massachusetts) : The MIT Press.

Tremblay, Gaëtan (2008), «Industries culturelles, économie créative et société de l'information» (p. 65-88), Global Media Journal, Volume 1, Issue 1.

Vénica, Catherine (2007), "Télécommunications mobiles avancées : réorganisation des filières et des logiques industrielles » (p. 45-64), in Bouquillion, Philippe ; Combès, Yolande (dir.), Les industries de la culture et de la communication en mutation, Paris : L'Harmattan (collection «Questions contemporaines»).

\section{RAPPORTS}

Centre National du Cinéma et de l'image animée $(\mathrm{CNC})$, « Le marché du jeu vidéo en $2009 »$, juin 2010 [en ligne],

http://www.cnc.fr/CNC_GALLERY_CONTENT/DOCUMENTS/publications/etudes/jeu_ video/Le_marche_jeu_video_180610.pdf, consulté le 15 mars 2011.

Ubisoft, «Document de référence 2010», juin 2010 [en ligne], http://www.ubisoftgroup.com/gallery_files/site/270/1042/2297.pdf, consulté le 04 avril 2011.

Vivendi, «Rapport annuel - Document de référence 2010 », mars 2011 [en ligne], http://www.vivendi.fr/vivendi/IMG/pdf/20100323_rapport_annuel_document_de_refere nce_2010.pdf, consulté le 04 avril 2011. 
